\title{
THE LOCAL MAGNETIC FIELD IN OUR GALAXY*
}

\author{
J.-L. HAN \\ Beijing Astronomical Observatory, Chinese Academy of Sciences, 100080, Beijing, China \\ and \\ G.-J. QIAO \\ United Laboratory for Optical Astronomy, Chinese Academy of Sciences and Department \\ of Geophysics, Beijing University, Beijing 100875, China
}

\begin{abstract}
The pitch angles of the local magnetic field in our Galaxy, previously derived from Rotation Measures (RMs) of pulsars by many authors, are not consistent with each other and with the pitch angles of the local spiral arms. That may be due to the fact that the used pulsar samples are located in different arms in which the directions of the magnetic fields are different. In this paper 2-D and 3-D models for the local magnetic field based on spiral arms are proposed for fitting the RMs of 129 nearby pulsars. In our models the amplitude of the uniform field changes sinusoidally to avoid abrupt reversals, and the directions are parallel or anti-parallel. The bestfitting 2-D model shows that in the Orion arm the strength of the regular field component is $2.4 \pm 0.3 \mu G$, with its direction towards $l=73^{\circ} \pm 3^{\circ}$. There is a direction reversal in SagittariusCarina arm beginning at $D_{r e v}=0.3 \mathrm{Kpc}$. The half "wavelength" of the sinusoidal variation is about $1.7 \pm 0.4 \mathrm{Kpc}$. The best fitting 3-D model shows that the scale height is only about 0.16 $\mathrm{Kpc}$, which means that the local uniform field is confined in the galactic plane. The strongest regular field in this 3-D model is about $2.8 \pm 0.3 \mu G$. The results from both, the 2-D model as well as the 3-D model, show that the orientation of the field is in excellent agreement with the spiral arms.
\end{abstract}

Key words: The Galaxy, Magnetic Field, Rotation Measure, Pulsars

\section{Introduction}

There are extensive studies of magnetic fields in spiral galaxies during the recent years. The general conclusion is that the regular fields have their strength at about several $\mu G$, and that the magnetic field lines follow more or less the spiral arms (e.g. Wielebinski, 1990; Beck, 1991).

In our Galaxy the existence of the magnetic field was deduced from the discovery of the linear polarization of starlight (Hall, 1949; Hiltner, 1949). The conclusion from reanalysing the data of stellar optical polarization is that the magnetic field is aligned with the galactic plane and in the direction of $l=45^{\circ}$ in the vicinity of the Sun, but beyond a circle of $600 \mathrm{pc}$ it is directed toward $l=70^{\circ}$ (Ellis and Aron, 1978). Simard-Normandin and Kronberg (1989) analysed the RMs of extragalactic radio sources and found that the local field point towards $l=76^{\circ}$ with reversals at the neighbouring arms. Sofue and Fujimoto (1983) found the bisymmetric magnetic field configuration could fit the RMs of extragalactic radio sources well. Inoue and Tabara (1981) concluded from a study of RMs of extragalactic sources and pulsars and optical polarization of starlight that $B_{\text {reg }}=1.6 \pm 0.4 \mu G$ and $l_{B}=100 \pm 10^{\circ}$ for the local field. There have been many attempts to derive the local or global magnetic field in our Galaxy from RMs of pulsars since the pioneering work of Manchester (1974). Because the pulsars have no intrinsic Faraday rotation, $\left\langle n_{e}\right\rangle$ along the lineof-sight can be estimated from Dispersion Measures (DMs). Pulsars are located

* The project was supported by NSF of China 
in best sites for measuring the average field and are really excellent probes of the galactic magnetic field. Manchester got the local field $B_{r e g}=2.2 \pm 0.4 \mu G$ and $l_{B}=94 \pm 11^{\circ}$ from the RMs of 28 pulsars. Thomson and Nelson (1980) used a more reasonable model to fit $\mathrm{RMs}$ of 48 pulsars and obtained $B_{\text {reg }}=3.5 \pm$ $0.3 \mu G, l_{B}=74 \pm 10^{\circ}$, with a reversal of the field at $D_{r e v}=170 \pm 90 p c$ towards the inner Galaxy. The scale height of the field is $70 \pm 40 p c$. Chi and Wolfendale (1990) analysed RMs of the 51 pulsars in solar vicinity and found that in the Orion arm, $B_{r e g}=1.0 \pm 0.4 \mu G, \quad l_{B}=80 \pm 19^{\circ}$, but between the Orion arm and Sagittarius arm, $B_{\text {reg }}=3.2 \pm 1.0 \mu G, l_{B}=237 \pm 14^{\circ}, \quad D_{r e v}=190 \pm 90 p c$.

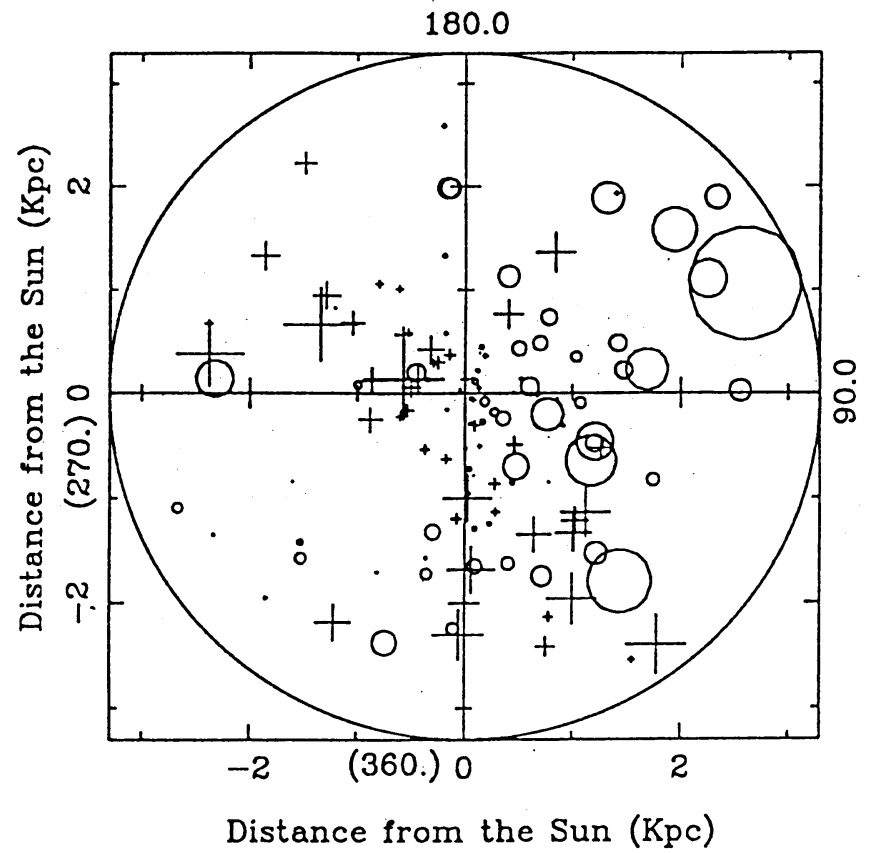

Fig. 1 The pulsars in our sample projected on the galactic plane. The values of RMs are proportional to the linear size of the symbols: positive values are indicated by + symbols, negative values by circles.

Rand and Kulkarni (1989) analysed the most complete RMs data of pulsars and found $B_{r e g}=1.6 \pm 0.2 \mu G, \quad L_{B}=96 \pm 4^{\circ}$, with a reversal at $D_{r e v}=600 \pm 80 p c$ towards the inner Galaxy. They also found that the concentric-ring field configuration fitted the data better than the bisymmetric one. Vallee $(1988,1991)$ found the pitch angles of magnetic field lines differ from those of the arms where they are located. In the Orion arm, the former is $16 \pm 4^{\circ}$, and the latter is $-18 \pm 3^{\circ}$. He agrees with Rand and Kukarni (1989) with the axisymmetric field configuration for our Galaxy, but not with regard to the concentric-ring model.

As we have seen above, the only possible general conclusion is that there is a reversal towards the inner Galaxy, but there is no agreement with regard to $D_{\text {rev }}$. The most important controversy we are concerned with is that why pitch angles of magnetic fields derived by different authors from RMs of pulsars differ from 
(2) Our results confirm that of Thomson and Nelson (1980) that the scalc height of the regular field is only about $0.1 \mathrm{Kpc}$. The local regular field is mainly confined in the galactic plane.

(3) The local field we got favours not Rand and Kulkarni's ring model (1989). In their ring model the field strength varies sinusoidally with the galactocentric radius; thus the pitch angle is zero. Our results with a pitch angle significantly distinct from zero would not support the ring model.

(4) Vallée's composed dynamo modes $m=0, n=0,1,2$ and $p=0$ can match the observational reversals well, however, if the field reverses in neighbouring arms, the global field has probably a bisymmetric configuration. According to the observed pattern of the local field and the spiral arms we are in favour of a bisymmetric global magnetic field in our Galaxy.

\section{Acknowledgements}

The project was supported in part by the Director Foundation of Beijing Astronomical Observatory for young astronomers. We also thank the support from NSF of China. We want to express our appreciation to Prof. Shou-Guan Wang and Prof. Fritz Krause for their suggestions and reading our manuscript carefully. The computations were performed with VAX and SUN in the Joined Laboratory for Radio Astronomy, Chinese Academy of Sciences.

\section{References}

Beck, R.: 1991, Proc. of IAU Symposium 144, 267.

Chi, X.: 1990, J. Phys. G. Nuch. Part. Phys. 16, 1049.

Costa, M.E., Meculloch, P.M., Hamilton, P.A.: 1991, M.N.R.A.S. 252, 13.

Ellis, R.S., Axon, D.J.: 1978, Astrophys. Space Sci. 54, 425.

Hall, J.S.: 1949, Science 109, 166.

Hamilton, P.N., Lyne, A.G.: 1987, M.N.R.A.S. 224, 1073.

Hiltner, W.A.: 1949, Ap. J. 109, 471.

Lynds, B.T.: 1980, A.J. 85(8), 1046.

Manchester, R.N.:1974, Ap.J. $188,637$.

Rand, R.J., Kulkarni, S.R.: 1989, A p.J. 343, 760.

Simard-Normandin, M., Kronberg, P.P.: 1980, Ap.J. 242, 74.

Thomson, R.C., Nelson, A.H.: 1980, M.N.R.A.S. 191, 863.

Vallée, J.P.: 1988, A.J. 95(3), 750.

Vallée, J.P.: 1991, Ap.J. 366, 450.

Wielebinski, R.: 1990, in The Interstellar Medium in Galaxies, 349. 